\title{
Miscibility in a degenerate fermionic mixture induced by linear coupling
}

\author{
Sadhan K. Adhikari \\ Instituto de Física Teórica, UNESP-São Paulo State University, 01.405-900 São Paulo, São Paulo, Brazil \\ Boris A. Malomed \\ Department of Interdisciplinary Studies, School of Electrical Engineering, Faculty of Engineering, Tel Aviv University, \\ Tel Aviv 69978, Israel \\ (Received 19 August 2006; published 22 November 2006)
}

\begin{abstract}
We consider a one-dimensional mean-field-hydrodynamic model of a two-component degenerate Fermi gas in an external trap, each component representing a spin state of the same atom. We demonstrate that the interconversion between them (linear coupling), imposed by a resonant electromagnetic wave, transforms the immiscible binary gas into a miscible state, if the coupling constant, $\kappa$, exceeds a critical value, $\kappa_{\mathrm{cr}}$. The effect is predicted in a variational approximation, and confirmed by numerical solutions. Unlike the recently studied model of a binary Bose-Einsten condensate with the linear coupling, the components in the immiscible phase of the binary fermion mixture never fill two separated domains with a wall between them, but rather form antilocked ( $\pi$-phase-shifted) density waves. Another difference from the bosonic mixture is spontaneous breaking of symmetry between the two components in terms of the numbers of atoms in them, $N_{1}$ and $N_{2}$. The latter effect is characterized by the parameter $\nu \equiv\left(N_{1}-N_{2}\right) /\left(N_{1}+N_{2}\right)$ (only $N_{1}+N_{2}$ is a conserved quantity), the onset of miscibility at $\kappa \geqslant \kappa_{\mathrm{cr}}$ meaning a transition to $\nu \equiv 0$. At $\kappa<\kappa_{\mathrm{cr}}, \nu$ features damped oscillations as a function of $\kappa$. We also briefly consider an asymmetric model, with a chemical-potential difference between the two components. The relation between the imbalance in the spin population, induced by the linear coupling, and the developing spatial structure resembles the known Larkin-Ovchinnikov-Fulde-Ferrell states in the Fermi mixture. Dynamical states, when $\kappa$ is suddenly switched from zero to a value exceeding $\kappa_{\mathrm{cr}}$, are considered too. In the latter case, the system features oscillatory relaxation to the mixed state.
\end{abstract}

DOI: 10.1103/PhysRevA.74.053620

PACS number(s): 03.75.Ss

\section{INTRODUCTION}

Experimental and theoretical studies of degenerate Bose [1] and Fermi [2] gases at ultra-low temperatures have become a vast research area. This field offers a unique testbed for the implementation of various ideas from classical and quantum nonlinear dynamics. In particular, experiments are possible in binary mixtures of atoms in two different hyperfine (spin) states. The mixtures were created by means of the sympathetic-cooling technique in the Bose-Einstein condensate (BEC) made of ${ }^{87} \mathrm{Rb}$ atoms [3]. In such a binary condensate, mutual interconversion, i.e., linear coupling between the two species, can be induced by means of a resonant (spin-flipping) electromagnetic wave, with a frequency in the $\mathrm{GHz}$ range [4]. Various effects were considered in this setting, including Josephson oscillations between the two states [5], domain walls between them [6], "breathing-together" oscillatory modes in the mixture [7], and nontopological vortices [8].

In a recent work [9], a different effect was predicted, namely, transition to miscibility induced by the linear coupling between the immiscible components in a binary condensate. It was demonstrated that, essentially, it occurs as a transition from a state with the two components separated by a domain wall to a completely mixed one. Quantitatively, the transition is characterized by an order parameter which determines the separation between centers of mass of the two components. With the increase of the linear-coupling strength (coupling constant), the order parameter decreases, and the full mixing sets in when it vanishes. Experimental observation of this effect is feasible due to the fact that the mixture of two hyperfine states in ${ }^{87} \mathrm{Rb}$ is immiscible, but close to the miscibility threshold $[3,10]$. The proximity of the mixture to the threshold can be additionally controlled by means of a Feshbach resonance, which can be induced by an external magnetic field [11] or optical pump [12].

Phase separation (demixing) due to the repulsion between two components was also investigated in boson-fermion mixtures [13], and, more recently, in a mixture of two fermion species $[14,15]$. In the latter case, a situation is possible when the species are two different spin states of the same fermion atom (as realized experimentally in ${ }^{40} \mathrm{~K}[16]$ and ${ }^{6} \mathrm{Li}$ [17]), hence the linear interconversion between them can be induced by the properly tuned electromagnetic wave.

In a recent experimental work [18], the population imbalance between two spin states ("polarization") in a degenerate ${ }^{6} \mathrm{Li}$ gas (trapped in a cigar-shaped cavity) was controlled by means of this technique. It was observed that, due to strong attraction between the different states (induced by the Feshbach resonance in external magnetic field) they formed a fully mixed state in the central part of the trap, that was separated by two sharp boundaries from side domains, filled with the atoms representing a majority state (see Figs. 3 and 5 in Ref. [18]).

Thus it is relevant to consider the transition to mixing in the binary fermionic gas under the action of the linear coupling (as well as in the bosonic mixture, the proximity to the miscibility point may be additionally controlled by means of the Feshbach resonance, which affects the strength of the repulsion between the two species [19]). This is the subject of the present work. 
Rigorous description of the dynamics of the degenerate gas of fermions is based on the system of quantummechanical equations of motion for individual fermions (see, e.g., Ref. [20]), which is difficult to handle if the gas contains many atoms. Simplified approaches of the mean-fieldhydrodynamic (MFHD) type were also elaborated for the description of quantum fermionic gases in static [14] and dynamical [15,21-23] settings. In the latter case, the approximation amounts to a single evolution equation for the fermion wave function, which formally resembles the GrossPitaevskii equation (GPE) for the boson gas [1], but features a different nonlinearity. This MFHD equation was successfully used to describe collapse [22] and solitons [23] in degenerate fermionic gases, and a system of nonlinearly coupled equations was employed to predict the phase separation in the binary gas with repulsion between the species [15].

In this work, we aim to investigate the onset of mixing in the immiscible binary fermion gas under the action of the extra linear coupling, in the (effectively) one-dimensional situation. In Sec. II, we formulate the model and then elaborate an analytical approach to the problem, based on a variational approximation (VA). The latter provides a description of the binary mixture in terms of the number-of-atoms ratio in the two components, $N_{1} / N_{2}$. In the presence of the linear mixing, only the total number is conserved, $N_{1}+N_{2}$, while the ratio $\nu=\left(N_{1}-N_{2}\right) /\left(N_{1}+N_{2}\right)$, if different from zero, characterizes spontaneous breaking of symmetry between the components. In fact, $\nu \neq 0$ is a specific, and most important, manifestation of the immiscibility in the fermionic system with the linear coupling (in the usual setting, without the linear interconversion, $N_{1}$ and $N_{2}$ are conserved separately, which makes the situation altogether different). The VA predicts the onset of mixing at a critical value of the coupling constant, $\kappa=\kappa_{\mathrm{cr}}$, with $\nu \equiv 0$ at $\kappa>\kappa_{\mathrm{cr}}$. The critical value is predicted as a a function of parameters measuring the total number of atoms and strength of the nonlinear repulsion between the species. Comparison with numerical results demonstrates that VA provides a qualitatively correct description of the transition to miscibility.

Basic numerical results are presented in Sec. III. They demonstrate a drastic difference of the phase separation in the fermionic mixture from the immiscible binary BEC: While in the boson gas the two species spontaneously separate and form a domain wall, with each component occupying the domain on either side of the wall (both in the ordinary setting, without the linear coupling [24], and when the coupling is switched on, up to the onset of the induced mixing [9]). A binary fermionic gas features the separation in an altogether different form: Each component forms a spatially modulated symmetric (even) density wave, which is antilocked, with the phase shift of $\pi$ between the components. A similar picture was predicted in the fermionic binary gas mixture without the linear coupling [15]. Here, a major difference is the above-mentioned overall spontaneous symmetry breaking between the components, manifested by $N_{1} / N_{2} \neq 1$ (in the immiscible bosonic condensate, the linear coupling does not break the equality between $N_{1}$ and $N_{2}$ [9]). As $\kappa$ approaches $\kappa_{\text {cr }}$ (the mixed state), we observe $N_{1} / N_{2}$ $\rightarrow 1$, while the modulation depth in the density waves decreases.
In Sec. IV, we briefly consider additional issues. One of them is the influence of asymmetry between the species, in the form of a chemical-potential difference between them, that can be induced by coupling of the atomic spin to an external dc magnetic field. Also considered is a dynamical relaxation of the binary gas into a mixed state, when $\kappa$ is suddenly switched from zero to a value exceeding $\kappa_{\mathrm{cr}}$. The results are summarized in Sec. V.

To conclude the Introduction, it is relevant to mention that the relation between the imbalance in the spin population, induced by the linear coupling, and the spatial densitymodulation waves developed by the two interacting species is similar to the known Larkin-Ovchinnikov-Fulde-Ferrell (LOFF) phase in a mixture of two fermion species with strongly differing values of the Fermi radius. As shown in original works [25] (see also the review in [26]), in that situation the pairing between the fermions belonging to the different species gives rise to Cooper pairs with a nonzero momentum, which translates into spatial structures in the form of crystalline patterns of the gap parameter. The LOFF phase has various implications for quark matter [26,27], and a possibility of its realization in the mixture of two different spin states in an ultracold fermion gas, with the interaction between them controlled via the Feshbach resonance, has been analyzed recently [28].

\section{THE MODEL AND VARIATIONAL APPROXIMATION}

\section{A. The mean-field-hydrodynamic equations}

Our starting point is the system of nonlinearly coupled one-dimensional MFHD equations for the fermion wave functions $\phi$ and $\psi$, as worked out in Ref. [15], to which we add, following Ref. [9] and references therein, the linearcoupling terms with strength $\kappa$ and, in the general case, a chemical-potential difference $\Delta \mu$ (in the larger part of the paper, we consider the symmetric system, with $\Delta \mu=0)$. In a scaled form, the equations are

$$
\begin{gathered}
i \phi_{t}=-\phi_{x x}+x^{2} \phi+\left(g_{1}|\phi|^{4 / 3}+g_{2}|\psi|^{2}\right) \phi-\kappa \psi, \\
i \psi_{t}=-\psi_{x x}+x^{2} \psi+\left(g_{1}|\psi|^{4 / 3}+g_{2}|\phi|^{2}\right) \psi-\kappa \phi+\Delta \mu \psi .
\end{gathered}
$$

Here, we imply the normalization

$$
N=\int_{-\infty}^{+\infty}\left[\Phi^{2}(x)+\Psi^{2}(x)\right] d x \equiv N_{1}+N_{2}=2 .
$$

In this notation the total number of atoms is hidden in the positive self-repulsion parameter [15] $g_{1}$, coefficient $g_{2}>0$ accounts for the nonlinear repulsion between the species, and the strength of the trapping potential (the coefficient in front of the parabolic potential $x^{2}$ ) is scaled to be 1 . The use of the same diagonal nonlinearity coefficient $g_{1}$ in both equations implies the same number of atoms in them and, unless we introduce $\kappa \neq 0$, the number of atoms will remain equal in both species. A characteristic feature of the MFHD approximation for the fermion gas is the power 4/3 of the nonlinear self-repulsive terms (recall the power is 2 in the ordinary GPE). 
In the particular case of $g_{1}=0$, Eqs. (1) and (2) admit a different interpretation, being tantamount to a system of GPEs for the binary BEC [9], in which the self-interaction coefficient (intraspecies scattering length) is set equal to zero by means of the Feshbach-resonance adjustment. This particular case establishes a link of the considered model to those for boson mixtures.

Coefficient $g_{2}$ in Eqs. (1) and (2), which accounts for the interspecies repulsion, can be controlled by means of the Feshbach-resonance technique in the fermion binary gas [19]. On the other hand, coefficient $g_{1}$ accounts for the Fermi pressure and cannot be altered by means of this technique. In the analysis presented below, we vary both coefficients $g_{1}$ and $g_{2}$, which corresponds to the normalization adopted in Eqs. (1) and (2) (in particular, varying $g_{1}$ actually implies the change in the number of atoms in the gas, as stated above).

We look for stationary solutions, $\{\psi, \phi\}$ $=\{\Psi(x), \Phi(x)\} e^{-i \mu t}$, with chemical potential $\mu$ and real functions $\Psi$ and $\Phi$ obeying the equations

$$
\begin{gathered}
\mu \Phi=-\Phi^{\prime \prime}+x^{2} \Phi+\left(g_{1} \Phi^{4 / 3}+g_{2} \Psi^{2}\right) \Phi-\kappa \Psi, \\
\mu \Psi=-\Psi^{\prime \prime}+x^{2} \Psi+\left(g_{1} \Psi^{4 / 3}+g_{2} \Phi^{2}\right) \Psi-\kappa \Phi+\Delta \mu \Psi
\end{gathered}
$$

(the prime stands for $d / d x$ ). Together with the normalization condition $\int_{-\infty}^{+\infty}\left[\Phi^{2}(x)+\Psi^{2}(x)\right] d x=2$, as per Eq. (3), Eqs. (4) and (5) can be derived as the variational equations $\delta L / \delta \Phi$ $=\delta L / \delta \Psi=\partial L / \partial \mu=0$ from the Lagrangian

$$
\begin{aligned}
L= & \mu+\frac{1}{2} \int_{-\infty}^{+\infty}\left[-\mu\left(\Phi^{2}+\Psi^{2}\right)+\left[\left(\Phi^{\prime}\right)^{2}+\left(\Psi^{\prime}\right)^{2}\right]\right. \\
& +x^{2}\left(\Phi^{2}+\Psi^{2}\right)+\frac{3}{5} g_{1}\left(\Phi^{10 / 3}+\Psi^{10 / 3}\right)+g_{2} \Phi^{2} \Psi^{2} \\
& \left.-2 \kappa \Phi \Psi+\frac{1}{2} \Delta \mu \Psi^{2}\right] d x .
\end{aligned}
$$

As said above, spontaneous symmetry breaking between the two species in the system with the linear coupling, where norms $N_{1}$ and $N_{2}$ are not conserved separately, can be characterized by the ratio

$$
\nu=\frac{N_{1}-N_{2}}{N_{1}+N_{2}} \equiv \frac{1}{2} \int_{-\infty}^{+\infty}\left[\Phi^{2}(x)-\Psi^{2}(x)\right] .
$$

In Eq. (7), the normalization of $N_{1}+N_{2}$ is taken into account as per Eq. (3). The objective of the analysis is to find $\nu$ as a function of $\kappa$ (for given $g_{1}$ and $g_{2}$ ), and thus identify the above-mentioned critical value, $\kappa_{\mathrm{cr}}$, past which (at $\kappa>\kappa_{\mathrm{cr}}$ ) only the symmetric solution exists, with $\nu \equiv 0$ [the symmetric solution exists at $\kappa<\kappa_{\text {cr }}$ too, but it is unstable (it does not represent the system's ground state) in that case; cf. a similar situation in the binary BEC with the linear coupling [9] ].

\section{B. Variational approximation}

The only tractable ansatz with which the VA can be applied to Lagrangian (6) is based on simple Gaussians,

$$
\Phi=a e^{-x^{2} / 2}, \quad \Psi=b e^{-x^{2} / 2},
$$

with constants $a$ and $b$. Normalization condition (3) for this ansatz takes the form of

$$
\sqrt{\pi}\left(a^{2}+b^{2}\right)=2,
$$

and the symmetry-breaking parameter, defined by Eq. (7), is

$$
\nu=(\sqrt{\pi} / 2)\left(a^{2}-b^{2}\right)
$$

Following the general method of the VA [29], we substitute ansatz (8) in Lagrangian (6) and perform the integration, which yields

$$
\begin{aligned}
\frac{L}{\sqrt{\pi}}= & \frac{\mu}{\sqrt{\pi}}+\frac{1-\mu}{2}\left(a^{2}+b^{2}\right)+\frac{3 \sqrt{15} g_{1}}{50}\left(a^{10 / 3}+b^{10 / 3}\right) \\
& +\frac{g_{2}}{2 \sqrt{2}}(a b)^{2}-\kappa a b .
\end{aligned}
$$

[normalization condition (9) can also be derived from this Lagrangian, as $\partial L / \partial \mu=0]$. In the framework of the VA, constants $a$ and $b$ are determined by equations $\partial L / \partial a=\partial L / \partial b$ $=0$, i.e.,

$$
\begin{aligned}
& \sqrt{\frac{3}{5}} g_{1} a^{7 / 3}+\frac{1}{\sqrt{2}} g_{2} a b^{2}-\kappa b=(\mu-1) a, \\
& \sqrt{\frac{3}{5}} g_{1} b^{7 / 3}+\frac{1}{\sqrt{2}} g_{2} b a^{2}-\kappa a=(\mu-1) b .
\end{aligned}
$$

Eliminating $\mu$ from these equations leads to a relation in which $(a-b)$ factorizes out, which yields an obvious symmetric solution, $a=b$. The remaining equation which determines the asymmetric solutions is

$$
\begin{gathered}
\sqrt{\frac{3}{5}} g_{1} a b\left(a^{2 / 3}+b^{2 / 3}\right)-\left(\frac{g_{2}}{\sqrt{2}} a b-\kappa\right) \\
\times\left[a^{4 / 3}+b^{4 / 3}+(a b)^{2 / 3}\right]=0 .
\end{gathered}
$$

Equations (9) and (12) constitute a system from which $a$ and $b$ should be found for given $g_{1}$ and $g_{2}$. Although this system cannot be solved in an analytical form, the critical value $\kappa_{\mathrm{cr}}$ can be easily found. Indeed, since only the symmetric solution exists at $\kappa \geqslant \kappa_{\mathrm{cr}}$, the respective bifurcation, at which the asymmetric solution merges into the symmetric one and disappears, occurs when Eqs. (9) and (12) themselves admit the symmetric solution $a=b=\pi^{-1 / 4}$. The substitution of this in Eq. (14) yields a simple result,

$$
\kappa_{\mathrm{cr}} \equiv \frac{g_{2}}{\sqrt{2 \pi}}-\frac{2 g_{1}}{\sqrt{15} \pi^{1 / 3}} .
$$

Note that Eq. (13) shows that $\kappa_{\text {cr }}$ vanishes at the point

$$
g_{2}=g_{2}^{\mathrm{cr}} \equiv 2 \sqrt{\frac{2}{15}} \pi^{1 / 6} g_{1} \approx 0.884 g_{1} .
$$

The meaning of the latter result is that, for $g_{2}<g_{2}^{\text {cr }}$, the VA predicts the binary fermionic gas to be in the mixed state even without the linear-coupling terms. 


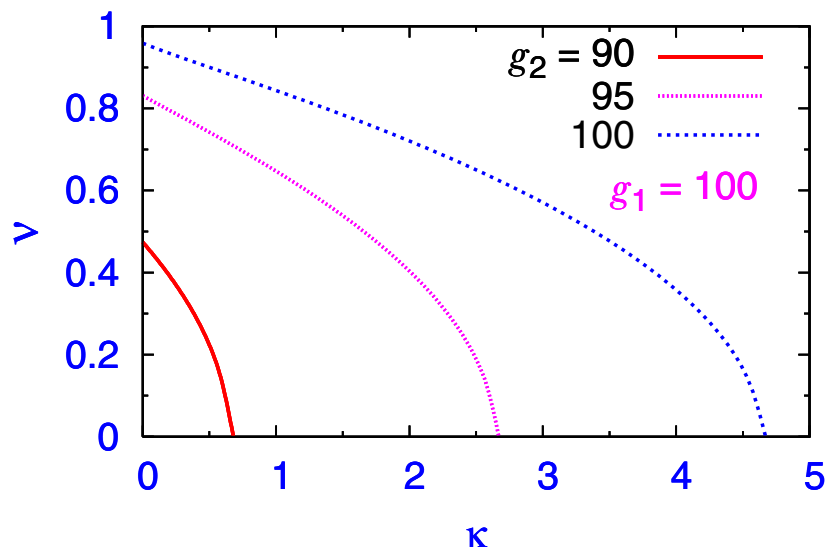

FIG. 1. (Color online) A set of bifurcation diagrams- $-\nu$ vs $\kappa$ plots-which show [in terms of the number-of-atoms symmetrybreaking parameter, $\nu$; see Eq. (10)] the asymmetric solution, as found from the variational equations (9) and (12) for a fixed $g_{1}$ and varying values of $g_{2}$. The solution with $\nu=0$ is an obvious symmetric one, $a=b=\pi^{-1 / 4}$; see text.

Close to the bifurcation which takes place at $\kappa=\kappa_{\mathrm{cr}}$, i.e., for $0<\Delta \kappa \equiv \kappa_{\mathrm{cr}}-\kappa \ll \kappa_{\mathrm{cr}}$, the system of equations (9) and (12) can be expanded, which yields the corresponding slightly asymmetric solution

$$
a=\pi^{-1 / 4} \pm \Delta a, \quad b=\pi^{-1 / 4} \mp \Delta a,
$$

where, in the first approximation,

$$
(\Delta a)^{2}=\Delta \kappa \frac{27 \sqrt{30}}{31 \sqrt{15} g_{2}-56 \sqrt{2} \pi^{1 / 6} g_{1}} .
$$

It is relevant to note that the condition necessary for the symmetry breaking (within the framework of the VA), $g_{2}$ $>g_{2}^{\text {cr }}$, see Eq. (14), guarantees that Eq. (16) yields $(\Delta a)^{2}$ $>0$ for $\Delta \kappa>0$, as it must be. Farther from the bifurcation point, Eqs. (9) and (12) can be solved numerically, which gives rise to a typical bifurcation picture displayed in Fig. 1, where we plot $\nu$ vs $\kappa$ for $g_{1}=100$ and $g_{2}=90,95$, and 100. In this case, the fermionic gas remains in the mixed state for $g_{2}<g_{2}^{\text {cr }} \approx 88.4$ even for $\kappa=0$. For $g_{2}>g_{2}^{\text {cr }}$, the two species separate, and the separation increases with growing $g_{2}$. Nevertheless, from Fig. 1 we find that, for $g_{2}=90$, 95, and 100, parameter $\nu$ vanishes for $\kappa>0.68,2.67$, and 4.67, respectively, and the system becomes mixed again. Comparison of further analytical predictions with numerical results is presented in detail in the next section.

\section{NUMERICAL RESULTS}

We solved coupled MFHD equations (1) and (2) numerically, using a real-time integration method based on the Crank-Nicholson discretization scheme, as elaborated in Ref. [30]. We discretize the mean-field equations, using time step 0.001 and space step 0.05 , in domain $-25<x<25$. We start with the Gaussian ground-state wave function of the linear harmonic oscillator as the input at $t=0, \Phi(x, t=0)=\Psi(x, t$ $=0)=\pi^{-1 / 4} \exp \left(-x^{2} / 2\right)$, while setting $g_{1}=g_{2}=0$. With these initial states, we have simulated the time evolution of Eqs. (1) and (2). In the course of the evolution, nonlinearity coefficients $g_{1}$ and $g_{2}$ are gradually switched on, at the rate of 0.0001 per time step of 0.001 . Solutions attain the final stationary configuration after the full nonlinearities and linear coupling $\kappa$ have been restored. All the wave functions displayed below are definitely stable against small perturbations, as stationary solutions to Eqs. (1) and (2).

With $\kappa=0$, the norms of the two components are conserved separately. In that case, we also used an imaginarytime-integration method based on the Crank-Nicholson discretization scheme, and the results obtained for Eqs. (1) and (2) are in agreement with the integration in real time, which verifies the correctness of the solutions. However, with $\kappa$ $\neq 0$, the normalizations of the two components are not separately conserved, and the imaginary-time-iteration method is not directly applicable.

A comparison of the analytical (variational) predictions from the previous section with numerical results can be made in terms of $\kappa_{\mathrm{cr}}$ as a function of $g_{2}-g_{2}^{\mathrm{cr}}$, for fixed values of $g_{1}$. This comparison is displayed in Fig. 2(a), where we plot variational and numerical results for $g_{1}=0,50,100$, and 200 . This plot demonstrates that the simple VA provides for a qualitatively reasonable prediction, provided that $g_{2}-g_{2}^{\mathrm{cr}}$ and $g_{1}$ are not too large.

A simple but noteworthy feature of the VA is the linear dependence of $g_{2}^{\text {cr }}$ on $g_{1}$; see Eq. (14). It can be compared to its numerically found counterpart, as shown in Fig. 2(b). The numerical dependence is nearly linear too, with the slope $d g_{2}^{\mathrm{cr}} / d g_{1} \approx 1.3$. The comparison with the analytical prediction for the slope, which is 0.884 as per Eq. (14), shows that the VA yields qualitatively correct, but not quite accurate, results. For $g_{1}=0$, the VA yields $g_{2}^{\mathrm{cr}}=0$, whereas the corresponding numerical result is $g_{2}^{\text {cr }} \approx 7$. This means that, even for binary atomic systems with no intraspecies interaction $\left(g_{1}=0\right)$, interspecies interaction $g_{2}$ has to be above the critical value $g_{2}^{\mathrm{cr}} \approx 7$ for the demixing to take place (which is a consequence of the fact that the two species can be mixed due to the pressure from the trapping potential). The simple VA fails to predict this fact, allowing for the demixing at any $g_{2}^{\mathrm{cr}}$.

Besides the conspicuous error in predicting the slope of the $g_{2}^{\mathrm{cr}}\left(g_{1}\right)$ dependence, another shortcoming of the VA is seen in the fact that the analytically predicted slope of the dependence of $\kappa_{\mathrm{cr}}$ vs $g_{2}-g_{2}^{\text {cr }}$ does not depend on $g_{1}$, while the numerically found slope changes with $g_{1}$ (in this connection, it is relevant to recall that the limited case of $g_{1}=0$, which is included in Fig. 2(a), corresponds, as explained above, not to the MFHD approximation for the fermion mixture, but rather to the system of coupled GPEs for the binary boson condensate with zero intraspecies interaction).

The VA could, in principle, be improved. An essential inaccuracy of simple ansatz (8) is the assumption that the wave functions monotonically decrease with $|x|$; in reality, numerically found wave functions of the ground state have several local minima and maxima; see Fig. 3. Even in the fully mixed state, the profile may be markedly different from the Gaussian, as suggested by the curves pertaining to $\kappa$ $=1.5$ in that figure. The simplest possibility to improve ansatz (8) in this respect would be to replace it by a generalized 

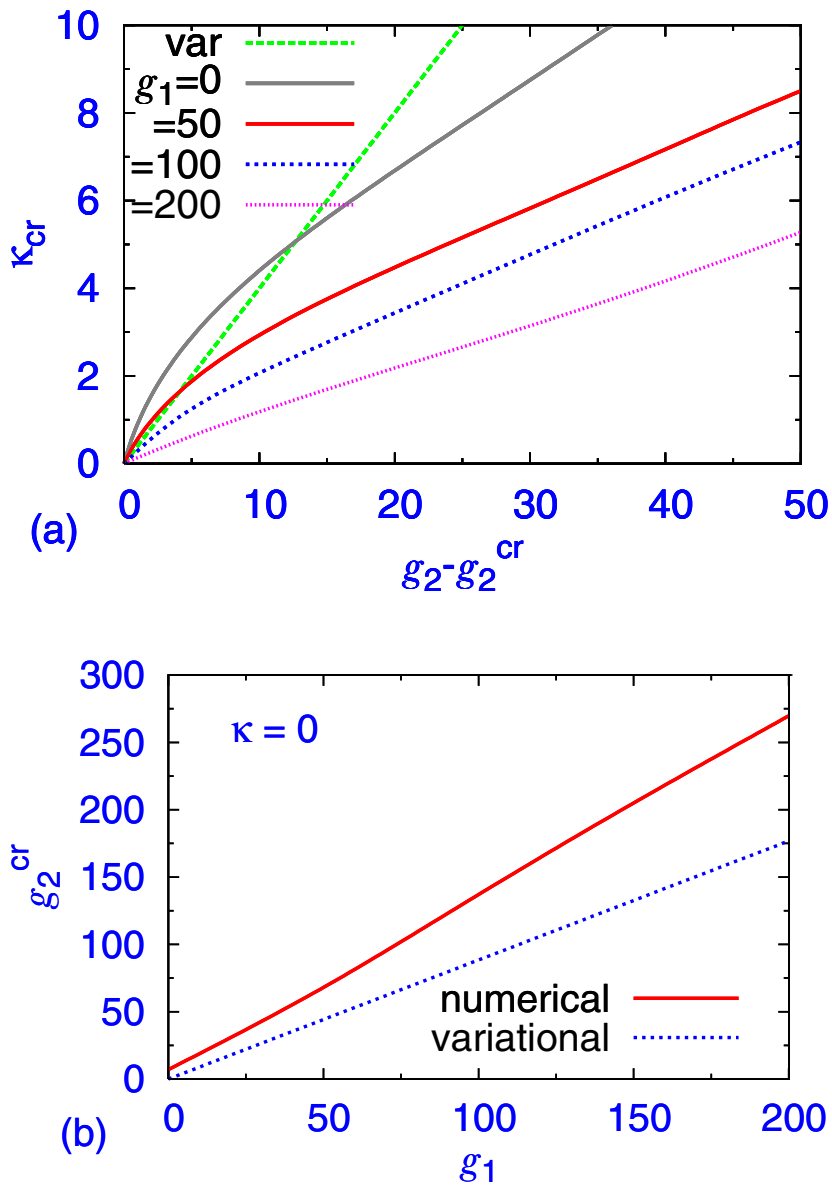

FIG. 2. (Color online) (a) The dependence of the critical value of the linear-coupling strength $\kappa_{\mathrm{cr}}$ on the nonlinear-repulsion constant $g_{2}$, plotted as $\kappa_{\text {cr }}$ vs $\left(g_{2}-g_{2}^{\text {cr }}\right)$ for $g_{1}=0,50,100$, and 200. Numerically obtained curves are labeled by respective values of $g_{1}$, while their counterpart produced by the variational approximation variational is labeled "var." Recall $g_{2}^{\text {cr }}$ is the value of $g_{2}$ below which the binary gas remains completely mixed in the absence of the linear coupling. (b) The numerically found dependence of $g_{2}^{\mathrm{cr}}\left(g_{1}\right)$, along with the respective variational prediction given by Eq. (14). The numerical dependence is (almost exactly) linear, although the slope 1.3 is different from its variational counterpart 0.884 .

one, $\Phi=\left(a_{0}+a_{2} x^{2}\right) e^{-x^{2} / 2}, \Psi=\left(b_{0}+b_{2} x^{2}\right) e^{-x^{2} / 2}$, with extra constants $a_{2}, b_{2}$. However, although the Lagrangian corresponding to the extended ansatz can be calculated in an analytical form, the variational equations following from it are so cumbersome that direct numerical solution of exact equations (1) and (2), or equivalently, Eqs. (4) and (5), is actually simpler.

Now we consider the results for the symmetric system $\Delta \mu=0$. Collecting the results for many values of $g_{1}$ and $g_{2}$, we have concluded that details of the solutions may differ considerably, but their qualitative properties are universal. An appropriate case to display generic results is one with $g_{1}=142$, and $g_{2}=100$ (in this case, $g_{2}^{\text {cr }}=137$ ). In Fig. 3, we display a set of ground-state wave functions found as $\kappa$ increases towards the transition to the full mixing at $\kappa=\kappa_{\mathrm{cr}}$ (in this case, $\kappa_{\mathrm{cr}} \approx 1.50$ ). A salient (and truly generic, as shown by comparison with a large pool of numerical data obtained
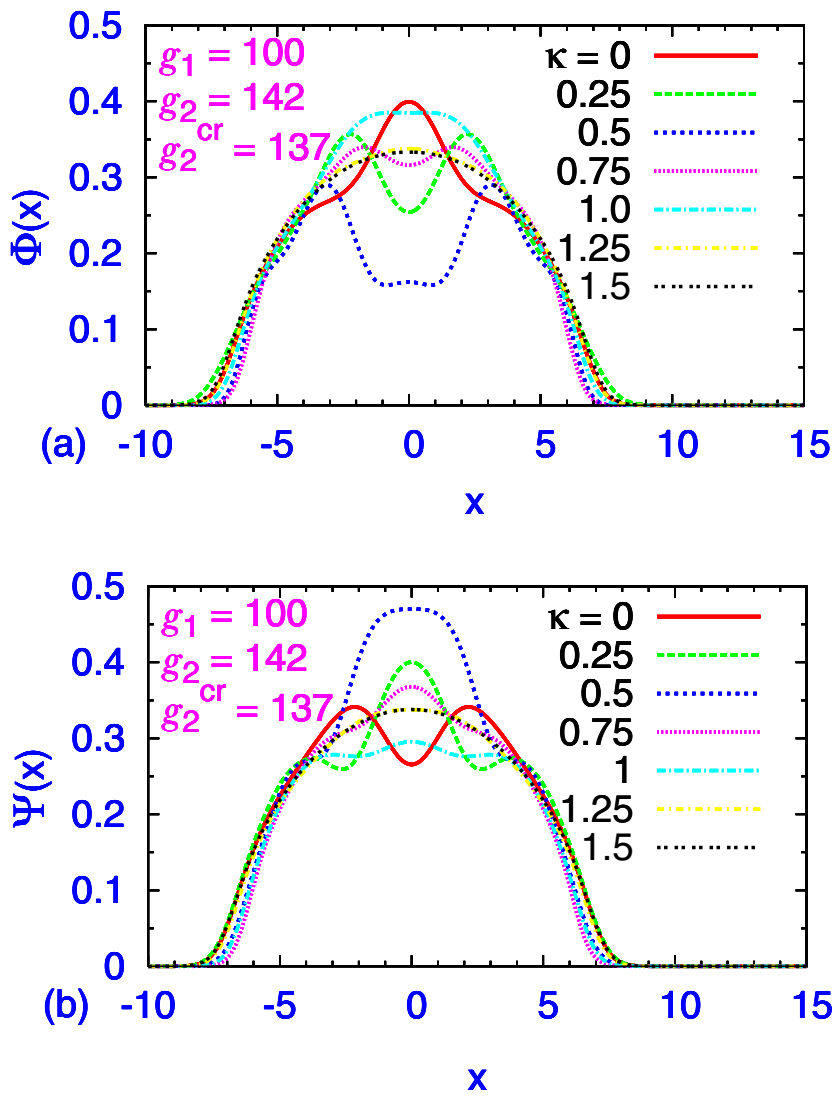

FIG. 3. (Color online) A generic example of a set of groundstate wave functions of the binary mixture $\Phi(x)$ and $\Psi(x)$ as obtained from the numerical solution of Eqs. (1) and (2) in the symmetric case $(\Delta \mu=0)$ for $g_{1}=100$ and $g_{2}=142$ and varying $\kappa$.

at other values of $g_{1}$ and $g_{2}$ ) feature of the ground state in the immiscible phase is that the two species do not collect themselves in different spatial domains, separated by a spontaneously formed wall (which is the case in the boson mixtures $[9,24]$ ), but, instead, they form two spatially modulated antilocked density waves (i.e., ones with a phase shift of $\pi$ ), while each wave remains an even function of $x$. In the absence of the linear coupling, a qualitatively similar structure of the ground state of the binary fermionic gas, with equal numbers of atoms in the two components, was reported in Ref. [15]. As $\kappa$ approaches the miscibility point, $\kappa_{\mathrm{cr}}$, the modulation in both components becomes more shallow, and finally completely disappears for $\kappa \geqslant \kappa_{\mathrm{cr}}$, where a full mixing between the components takes place.

As overall characteristics of the immiscible state, we use the number-of-atoms symmetry-breaking parameter, defined by Eq. (7), and, in addition,

$$
\Xi \equiv \frac{\int_{-\infty}^{+\infty} x^{2}\left[\Phi^{2}(x)-\Psi^{2}(x)\right] d x}{\int_{-\infty}^{+\infty} x^{2}\left[\Phi^{2}(x)+\Psi^{2}(x)\right] d x},
$$

which quantifies the antilocking between the modulated density waves in the two components. For the same typical case 


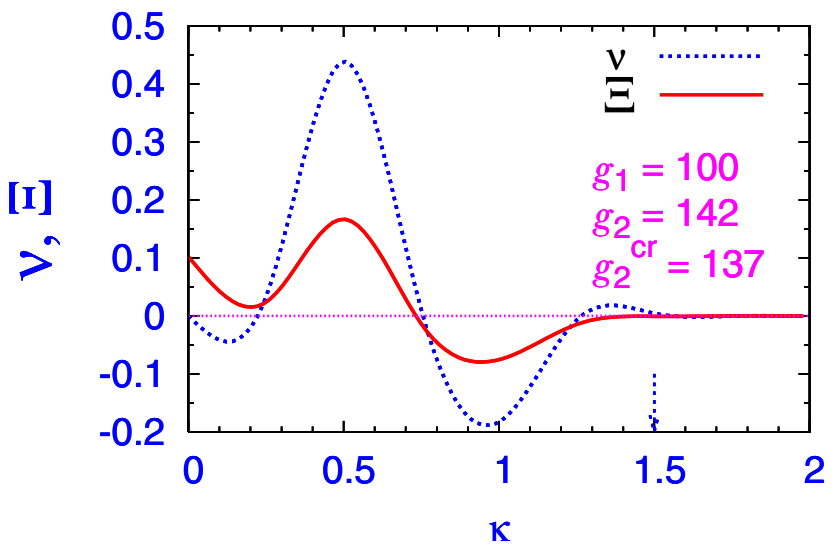

FIG. 4. (Color online) Parameters which characterize the spontaneously established inequality in numbers of atoms between the linearly coupled immiscible species $\nu$ [see Eq. (7)] and antilocking in the spatial density modulation of the species $\Xi$ [see Eq. (17)] are shown as functions of $\kappa$, found from the numerical solution of Eqs. (1) and (2) in the symmetric case $(\Delta \mu=0)$. The critical value $\kappa_{\mathrm{cr}}$ $\approx 1.50$ of $\kappa$ above which complete mixing takes place in this case is shown by an arrow.

which is illustrated by the set of ground-state profiles in Fig. $3, \nu$ and $\Xi$ are displayed, as functions of $\kappa$, in Fig. 4. A salient feature of the dependences (which is another truly generic feature, observed in all other cases for which numerical solutions were obtained) is the oscillatory character of the decay of both $\nu$ and $\Xi$, observed as $\kappa$ approaches $\kappa_{\mathrm{cr}}$. Naturally, we have $\nu=0$ at $\kappa=0$ (we always started the computation from a symmetric state, with $N_{1}=N_{2}$, at $\kappa=0$, when the two numbers of atoms are conserved separately), while $\Xi(\kappa=0)$ is different from zero, because, as mentioned above, the antilocking between the modulated density waves in the two components of the ground state (i.e., demixing in this state) takes place also in the system without the linear interconversion [15]. Because the symmetry breaking between the two components is spontaneous, the overall signs of $\nu$ and $\Xi$ are, of course, selected arbitrarily. The plots in Fig. 4 are generated by choosing $\Xi(\kappa=0)>0$, and then drawing the curves by continuity.

Another feature that deserves special consideration is that, at some values of $\kappa$ (in Fig. 4, these are $\kappa \approx 0.25$ and $\kappa$ $\approx 0.75$ ), both $\nu$ and $\Xi$ take nearly zero values almost simultaneously, suggesting that the system falls into a nearly mixed state at these points. Indeed, the consideration of the profiles in a vicinity of one of these points, see Fig. 5, shows that the two profiles are almost identical at $\kappa$ close to 0.24 ; nevertheless, the modulation does not completely disappear in this case, and the situation appears to be an accidental mixing. As $\kappa$ moves away from this point, both $\nu$ and $\Xi$ become different from zero, which implies restoration of the demixing and difference in numbers of atoms in the two components, as seen in Fig. 5.

It is noteworthy that the deepest antilocked modulation in the two components (in other words, a maximum of $|\Xi(\kappa)|$ ) is attained not at $\kappa=0$, but at a finite value of the coupling constant. As seen from Figs. 3 and 4, in the present example this value is $\kappa \approx 0.5$.
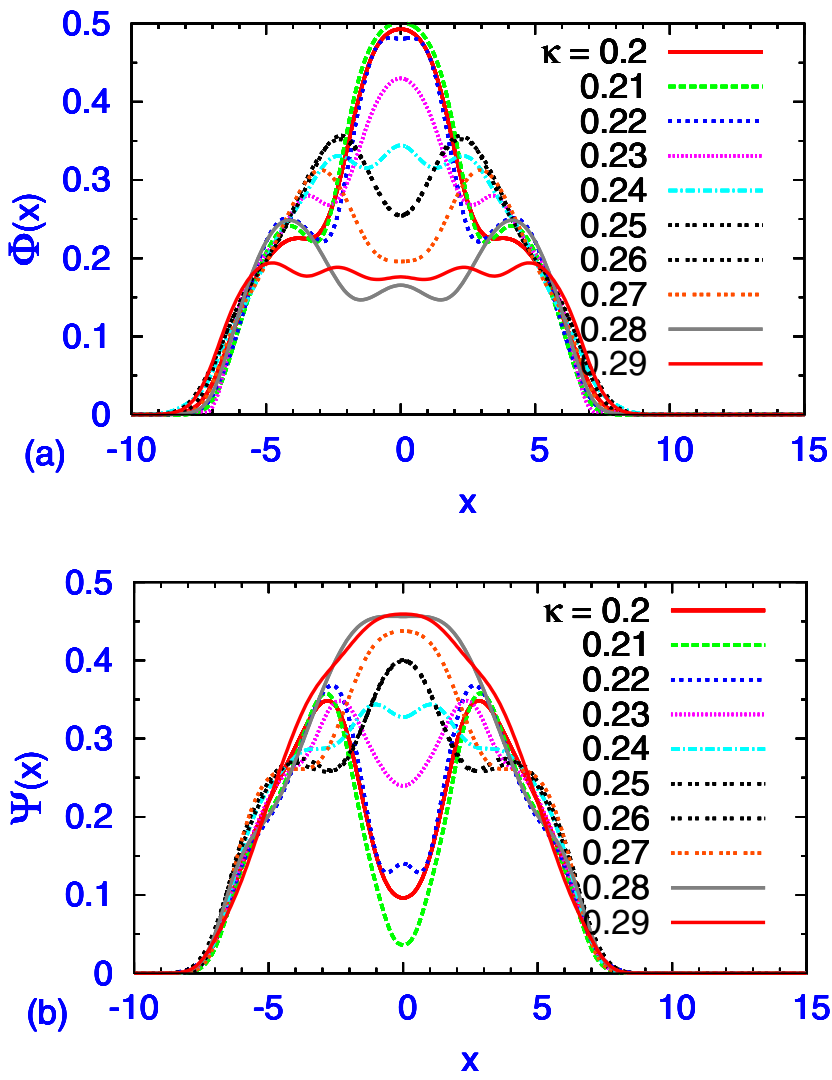

FIG. 5. (Color online) In addition to Fig. 3, an extra set of profiles of the wave functions in the symmetric case $(\Delta \mu=0)$, for $g_{1}=100$ and $g_{2}=142$, in the ground state around $\kappa=0.25$, where nearly complete mixing occurs accidentally.

\section{FURTHER RESULTS}

\section{A. The asymmetric system $(\Delta \mu \neq 0)$}

We now proceed to the role of the chemical-potential asymmetry in Eqs. (1) and (2). If the asymmetry is present in Eq. (2), $\Delta \mu \neq 0$, the inequality of the numbers of atoms in the asymmetric components is not spontaneous (i.e., the sign of $\nu$ is not selected arbitrarily), unlike the symmetric system: For $\Delta \mu>0, N_{1}$ is larger than $N_{2}$ (similar to what was found in the model of the asymmetric boson mixture [9]). Accordingly, $\Delta \mu$ tends to suppress the oscillations in dependences $\nu(\kappa)$ and $\Xi(\kappa)$, which were a salient feature of the symmetric system (see Fig. 4). Our results clearly show (Fig. 7) that, in the model with a nonzero difference in the chemical potentials, $N_{1}$ and $N_{2}$ are never exactly equal.

In Fig. 6, we display a counterpart of Figs. 3 and 5, i.e., a set of profiles of the wave functions in the ground state for $g_{1}=100$ and $g_{2}=142$, in the presence of relatively strong asymmetry, with $\Delta \mu=0.4$. Some residual oscillations in the shape of the wave functions are still seen, but they are much less pronounced than in Figs. 3 and 5.

A counterpart of Fig. 4 for the same example of the asymmetric system is displayed in Fig. 7, where we plot $\nu$ and $\Xi$ vs $\kappa$ for the asymmetric fermion mixture studied in Fig. 6. As expected, the figure shows that the oscillations in the plots of $\nu(\kappa)$ and $\Xi(\kappa)$ are greatly reduced and, on the contrary to the 

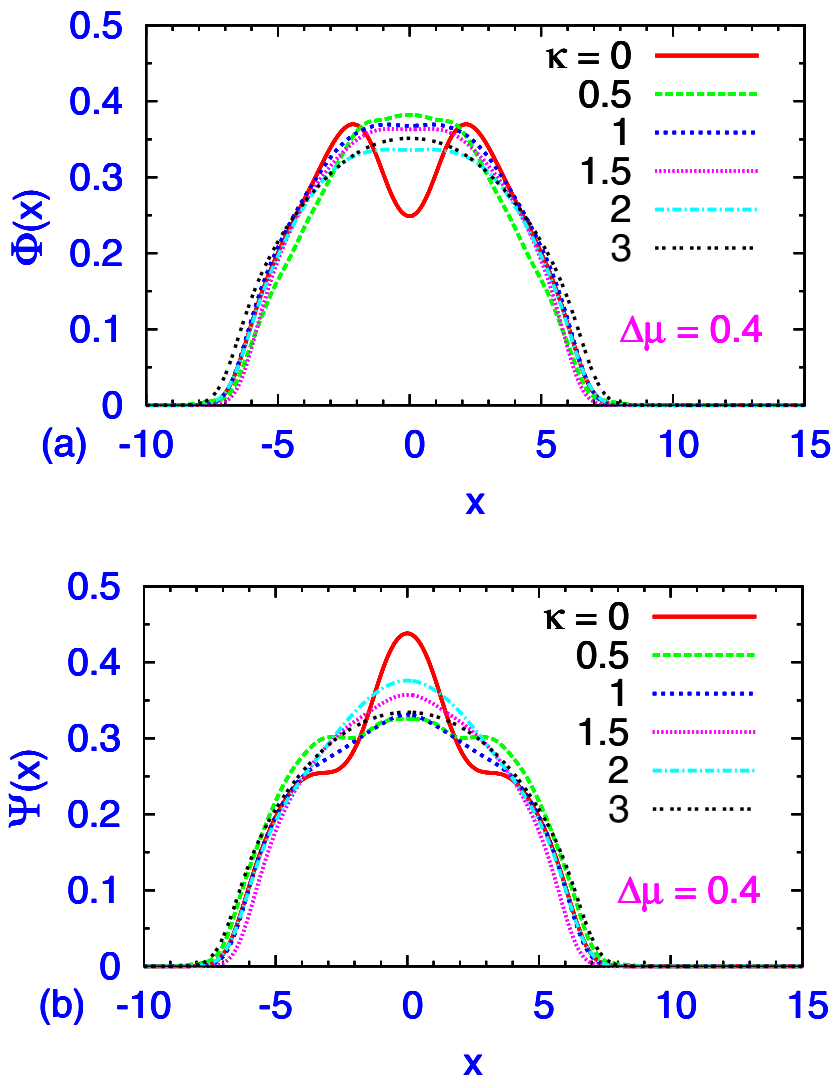

FIG. 6. (Color online) A generic example of a set of groundstate wave functions of the binary mixture $\Phi(x)$ and $\Psi(x)$ for different $\kappa$, as obtained from numerical solution of Eqs. (1) and (2) in the asymmetric model, with $g_{1}=100, g_{2}=142$, and $\Delta \mu=0.4$.

symmetric model (see Fig. 4), there is no point at which these functions, $\nu(k)$ and $\Xi(k)$, would vanish; instead, they decay asymptotically at large values of $\kappa$. The nonvanishing of $\nu(k)$ implies that the numbers of atoms in the two components never become exactly equal at $\Delta \mu \neq 0$.

\section{B. Dynamical relaxation}

The above analysis was based on solutions of Eqs. (1) and (2) [the stability of the solutions was verified also within the

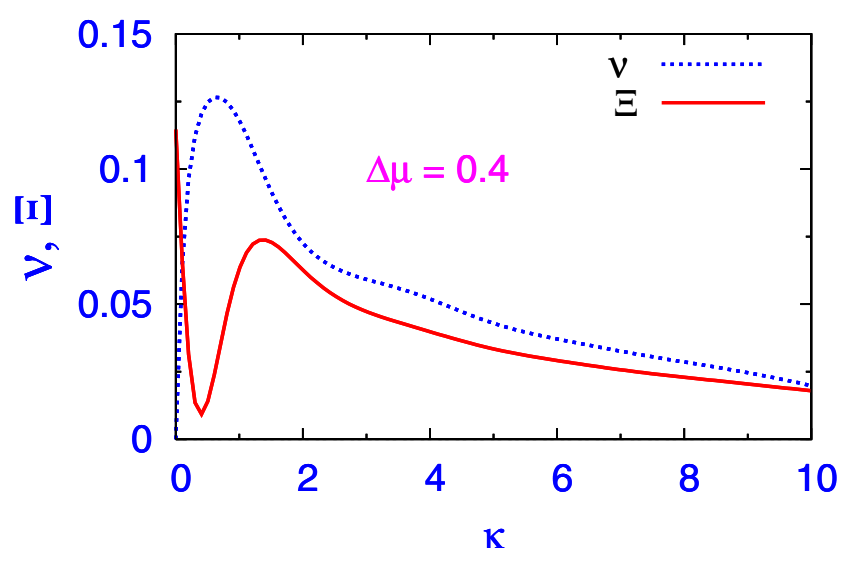

FIG. 7. (Color online) The same as in Fig. 4, but for the asymmetric system corresponding to Fig. 6, i.e., with $\Delta \mu=0.4$ in Eq. (2).

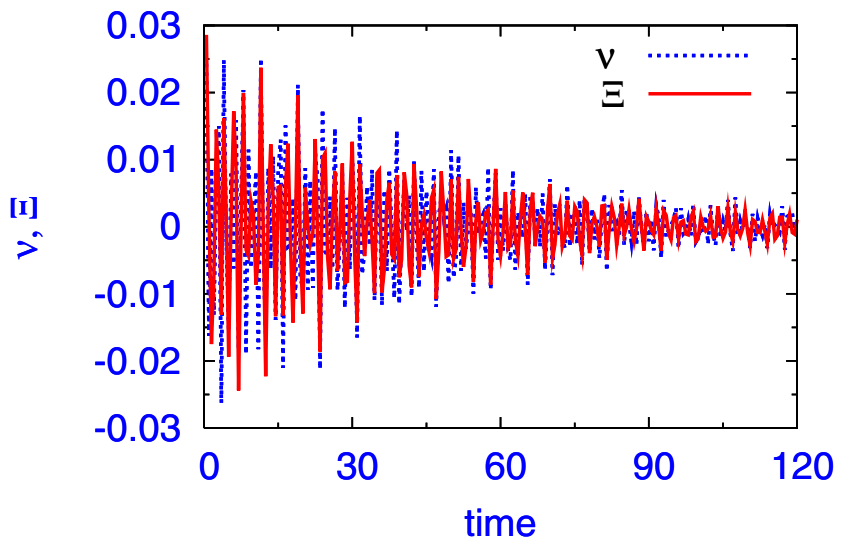

FIG. 8. (Color online) Relaxation of the initial immiscible state in the system with $g_{1}=100, g_{2}=140$, and $\kappa=0$ into a mixed state, after $\kappa$ was suddenly switched on from 0 to 4 (recall that $\kappa_{\mathrm{cr}} \approx 1.5$ for these values of $g_{1}$ and $g_{2}$ ).

framework of the full time-dependent MFHD equations (1) and (2)]. Another aspect of the immiscibility/miscibility transition is relaxation of an initial immiscible state after the coupling terms were suddenly switched on. In the context of the boson mixture, a similar problem was considered in Ref. [9]. In that work, it was found that the sudden application of the strong linear coupling did not lead to relaxation of the system into a mixed state, but rather to persistent nonequilibrium oscillations. Here, we performed numerical experiments, starting from the phase-separated state at $\kappa=0$ and suddenly adding the linear coupling between the components, with $\kappa>\kappa_{\mathrm{cr}}$ (in the symmetric system, with $\Delta \mu=0$ ). The result can be adequately presented in the form of the time dependence of the immiscibility parameters, $\nu$ and $\Xi$, that were defined above [see Eqs. (7) and (17)]. A typical example of this dependence is displayed in Fig. 8, which shows oscillatory relaxation to the mixed state characterized by $\nu=\Xi=0$.

\section{CONCLUSION}

We have demonstrated that the linear coupling between two different spin states (induced by a resonant electromagnetic field), which form an immiscible binary fermionic gas, may enforce a transition to miscibility. The description of the degenerate gas is based on a system of equations for the wave functions of the two components, derived in the MFHD (mean-field-hydrodynamic) approximation. The transition to the induced miscibility was predicted by means of the variational approximation, and then verified in numerical computations. In contrast with the induced onset of miscibility in the bosonic mixture [9], in the present case the immiscible components do not form a domain wall, separately filling two domains partitioned by the wall. Instead, they form a pair of antilocked ( $\pi$-phase-shifted) spatially modulated density waves, and spontaneously break the equality of numbers of atoms in the two components (the latter feature was not observed in the bosonic mixture). The relation between the imbalance in the spin population, induced by the linear cou- 
pling, and the spatial density-wave patterns developed by the species is similar to the LOFF phase in a binary fermion gas with unequal values of the Fermi radius [25-28]. As the linear-coupling strength $\kappa$ approaches the inducedmiscibility threshold $\kappa_{\mathrm{cr}}$, the spatial modulation in the density waves vanishes and the numbers of atoms in the components become equal. We have also considered the influence of inherent asymmetry between the components (the chemical-potential difference between them) and dynamical situations, when $\kappa$ is suddenly switched on (in the symmetric system) from zero to a value exceeding $\kappa_{\mathrm{cr}}$. In the latter case, the system features oscillatory relaxation to the mixed state.

\section{ACKNOWLEDGMENTS}

The work of B.A.M. was supported, in part, by the Israel Science Foundation through the Grant No. 8006/03. The work of S.K.A. was supported in part by the FAPESP and CNPq of Brazil.
[1] C. Pethick and H. Smith, Bose-Einstein Condensation in Dilute Gases (Cambridge University Press, Cambridge, UK, 2002); L. P. Pitaevskii and S. Stringari, Bose-Einstein Condensation (Clarendon Press, Oxford and New York, 2003).

[2] A. Minguzzi, S. Succi, F. Toschi, M. P. Tosi, and P. Vignolo, Phys. Rep. 395, 223 (2004); Q. J. Chen, J. Stajic, S. Tan, and K. Levin, ibid. 412, 1 (2005).

[3] C. J. Myatt, E. A. Burt, R. W. Ghrist, E. A. Cornell, and C. E. Wieman, Phys. Rev. Lett. 78, 586 (1997).

[4] R. J. Ballagh, K. Burnett, and T. F. Scott, Phys. Rev. Lett. 78, 1607 (1997).

[5] J. Williams, R. Walser, J. Cooper, E. Cornell, and M. Holland, Phys. Rev. A 59, R31 (1999); P. Öhberg and S. Stenholm, ibid. 59, 3890 (1999).

[6] D. T. Son and M. A. Stephanov, Phys. Rev. A 65, 063621 (2002)

[7] S. D. Jenkins and T. A. B. Kennedy, Phys. Rev. A 68, 053607 (2003).

[8] Q.-H. Park and J. H. Eberly, Phys. Rev. A 70, 021602(R) (2004).

[9] I. M. Merhasin, B. A. Malomed, and R. Driben, J. Phys. B 38, 877 (2005).

[10] H. Pu and N. P. Bigelow, Phys. Rev. Lett. 80, 1130 (1998); D. S. Hall, M. R. Matthews, J. R. Ensher, C. E. Wieman, and E. A. Cornell, ibid. 81, 1539 (1998).

[11] S. Inouye, M. R. Andrews, J. Stenger, H. J. Miesner, D. M. Stamper-Kurn, and W. Ketterle, Nature (London) 392, 151 (1998); P. Timmermans, P. Tommasini, M. Hussein, and A. Kerman, Phys. Rep. 315, 199 (1999).

[12] P. O. Fedichev, Yu. Kagan, G. V. Shlyapnikov, and J. T. M. Walraven, Phys. Rev. Lett. 77, 2913 (1996); M. Theis, G. Thalhammer, K. Winkler, M. Hellwig, G. Ruff, R. Grimm, and J. H. Denschlag, ibid. 93, 123001 (2004).

[13] K. Molmer, Phys. Rev. Lett. 80, 1804 (1998); P. Capuzzi, A. Minguzzi, and M. P. Tosi, Phys. Rev. A 68, 033605 (2003); Z. Akdeniz, A. Minguzzi, P. Vignolo, and M. P. Tosi, Phys. Lett. A 331, 258 (2004); Y. Takeuchi and H. Mori, Phys. Rev. A 72, 063617 (2005); M. Modugno, F. Ferlaino, F. Riboli, G. Roati, G. Modugno, and M. Inguscio, ibid. 68, 043626 (2003); M. Zaccanti, C. D’Errico, F. Ferlaino, G. Roati, M. Inguscio, and G. Modugno, ibid. 74, 041605(R) (2006).

[14] A. Amoruso, I. Meccoli, A. Minguzzi, and M. P. Tosi, Eur.
Phys. J. D 8, 361 (2000); R. Roth and H. Feldmeier, J. Phys. B 34, 4629 (2001).

[15] S. K. Adhikari, Phys. Rev. A 73, 043619 (2006).

[16] B. DeMarco and D. S. Jin, Science 285, 1703 (1999).

[17] K. M. O'Hara, S. L. Hemmer, M. E. Gehm, S. R. Granade, and J. E. Thomas, Science 298, 2179 (2002); K. E. Strecker, G. B. Partridge, and R. G. Hulet, Phys. Rev. Lett. 91, 080406 (2003).

[18] G. B. Partridge, W. Li, Y. A. Liao, R. G. Hulet, M. Haque, and H. T. C. Stoof, Phys. Rev. Lett. (to be published), e-print condmat/0608455.

[19] K. M. O’Hara, S. L. Hemmer, S. R. Granade, M. E. Gehm, J. E. Thomas, V. Venturi, E. Tiesinga, and C. J. Williams, Phys. Rev. A 66, 041401(R) (2002); K. Dieckmann, C. A. Stan, S. Gupta, Z. Hadzibabic, C. H. Schunck, and W. Ketterle, Phys. Rev. Lett. 89, 203201 (2002); T. Loftus, C. A. Regal, C. Ticknor, J. L. Bohn, and D. S. Jin, ibid. 88, 173201 (2002); C. A. Regal, M. Greiner, and D. S. Jin, ibid. 92, 083201 (2004).

[20] M. Salerno, Phys. Rev. A 72, 063602 (2005).

[21] P. Capuzzi, A. Minguzzi, and M. P. Tosi, Phys. Rev. A 67, 053605 (2003).

[22] S. K. Adhikari, Phys. Rev. A 70, 043617 (2004).

[23] S. K. Adhikari, Phys. Rev. A 72, 053608 (2005); J. Phys. B 38, 3607 (2005); Laser Phys. Lett. 3, 605 (2006); Eur. Phys. J. D 40, 157 (2005).

[24] M. Trippenbach, K. Goral, K. Rzazewski, B. Malomed, and Y. B. Band, J. Phys. B 33, 4017 (2000).

[25] A. I. Larkin and Yu. N. Ovchinnikov, Zh. Eksp. Teor. Fiz. 47, 1136 (1964) [Sov. Phys. JETP 20, 762 (1965)]; P. Fulde and R. A. Ferrell, Phys. Rev. 135, A550 (1964).

[26] R. Casalbuoni and G. Nardulli, Rev. Mod. Phys. 76, 263 (2004).

[27] M. Alford, J. A. Bowers, and K. Rajagopal, Phys. Rev. D 63, 074016 (2001)

[28] D. E. Sheehy and L. Radzihovsky, Phys. Rev. Lett. 96, 060401 (2006); L. He, M. Jin, and P. Zhuang, Phys. Rev. B 73, 214527 (2006).

[29] B. A. Malomed, in Progress in Optics, edited by E. Wolf (North-Holland, Amsterdam, 2002), Vol. 43, p. 71.

[30] S. K. Adhikari and P. Muruganandam, J. Phys. B 35, 2831 (2002); P. Muruganandam and S. K. Adhikari, ibid. 36, 2501 (2003). 